\title{
A personal experience of the 5:2 diet
}

\begin{abstract}
Growing up I participated in sports regularly and was generally active on a daily basis When in my thirties as the career accelerated and family life took up the remaining time, there was little room for physical activity and weight gain set in. Probably a history that many recognize. In my late forties I was between 95 and $100 \mathrm{~kg}$ with a BMI of 27-30. I was trying to stay active and at work we would go for a run of $5-7 \mathrm{~km}$ once or twice a week. Performance was poor and recovery took more than a day. When I heard of the 5:2 diet and saw the BBC program I immediately wanted to try this and started on the diet in early April 2013. Within two weeks I was able to run longer at a better pace and recovery was much faster. This was before any measurable weight loss On a regular $7 \mathrm{~km}$ route $\mathrm{I}$ went from $6 \mathrm{~min}$ pr $\mathrm{km}$ to $5 \mathrm{~min}$ pr $\mathrm{km}$ within 5 months. The weight loss was much slower but over the first year I lost $10 \mathrm{~kg}$ and over the following half year another $5 \mathrm{~kg}$ to achieve a BMI below 25. The fast improvement in exercise performance implies that very early on there was improvement of hormonal responses in liver and fat depots making stored energy more accessible. This was supported by a much faster recovery and general feeling of higher energy level and wellness.
\end{abstract}

Volume 6 Issue 5 - 2017

Torben Österlund

Torn Pharma Consulting, Sweden

Correspondence: Torben Österlund, Torn Pharma Consulting, Orkestervägen 28, 22472 Lund, Sweden. Tel +46 736407 793, Email toosathome@yahoo.se

Received: April 10, 2017| Published: May 03, 2017

\section{The 5:2 diet initial phase}

I think many will recognize that while you were generally more active growing up, by the end of your thirties having family and career taking all time, there was little room for exercise (unless you had a physically demanding job). From the age of 32 to 40 I gained more than $15 \mathrm{~kg}$ and was generally overweight and had little time for physical activity. For many years I tried to regain some fitness by various types of exercise without any significant progress. At my former workplace there were good opportunities to participate in various physical activities for better fitness and I went running together with colleagues 5 -6times per month, usually on a $5 \mathrm{~km}$ round. This took roughly $30-35 \mathrm{~min}$ and recovery after such exercise was usually a day or two. The latter meant that I would have sour legs and not be keen to run again in that period.

In the spring of 2013 I saw the BBC documentary by Michael Mosley on diet induced lowering of IGF-1 and possibility to change the opportunities for exercise and helping the body to go into a more preserving mode. ${ }^{1}$ The program suggested a 5:2 diet where 2 days a week the caloric intake would be $25 \%$ or less than the normal daily intake. Compared to all previously suggested diets this seemed much more feasible to follow over a long period of time. Two days a week you would eat very little but all other days you could eat normally making it easier to mentally cope with the hunger signals on the fasting days, knowing that the following day you could eat whatever you like.

Early April 2013 I started following the 5:2 diet usually fasting on Mondays and Thursdays. The first couple of fasting days were very strange to the body and I had strong hunger signals at meal times just as expected, but generally it was easier than anticipated to follow the diet without emotional downsides. At first I was reluctant to exercise on fasting days but after 3 weeks I tried it for the first time with fine result and since then I actually prefer to do exercise on fasting days, probably since the access to the energy stores is better on those days (lower insulin levels). Within two weeks of starting I experienced a strong improvement in performance during the runs. I swished to $7 \mathrm{~km}$ and increased the pace for almost every time, doing the round in
$41 \mathrm{~min}$ in mid-April and 35min in mid-September (Figure 1). In this period I lost less than $5 \mathrm{~kg}$. The weight reduction was much slower than the improvement in exercise performance, but within one year I had lost some $10 \mathrm{~kg}$. I lost another $5 \mathrm{~kg}$ the following half year and ended close to $82 \mathrm{~kg}$ on average (spanning from 80 to $85 \mathrm{~kg}$ ) which became my new steady state level (probably still gaining muscle mass while losing a little of fat).

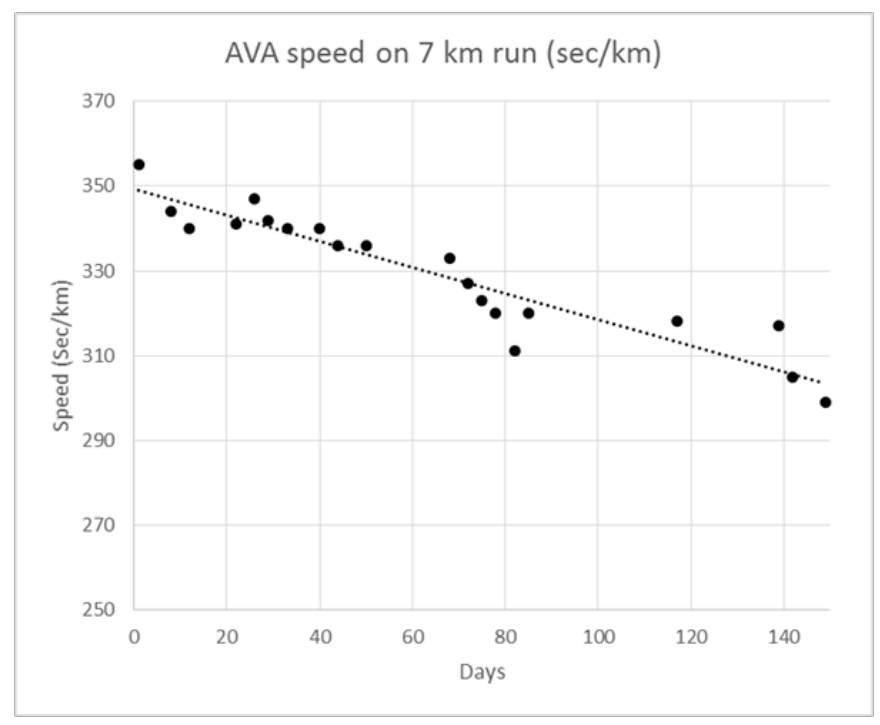

Figure I The progress of running speed on the same basically flat $7 \mathrm{~km}$ round in the first 5month after starting on the 5:2 diet. The speed/pace is given as seconds' pr km on average for the whole round.

\section{Long term performance}

After the initial improvement in running performance I have slowly gone into more long distance running (particular trail-running (aiming for ultras), orienteering and the occasional half-marathon). This supplemented with gymnastics and weight training. Thus, I am no longer improving my pace but on the distances and on hill-running. I have used an exercise app to keep track of distances and time spends 
on exercise. From May to December 2013 I averaged $4 \mathrm{~h}$ exercise pr month but improved much on pace and ability to run somewhat longer at times. In 2014 and $2015 \mathrm{I}$ jumped to more than $11 \mathrm{~h}$ a month and in 2016 this jumped further to more than $15 \mathrm{~h}$ per month. The best month in the whole period on 5:2 diet I have been above $20 \mathrm{~h}$ of exercise. I have also run more than $140 \mathrm{~km}$ in one month at best, averaging a little over $100 \mathrm{~km}$ a month over the whole of 2016 . This is of cause far behind many of the more experienced runners in particular trailand ultra-runners, so there is room for improvement. However, even contemplating to do this kind of long distance running with hills and obstacles would not have been possible without the 5:2 diet, despite my youth history of doing sports. I have not made any changes to the diet itself since this was already diverse and generally healthy. All changes were a result of the diet and the exercise changes that followed.

One peculiar observation I made is that in the late evenings (usually at or after normal bedtime) I experience an increase in body-heat (I feel warmer but not feverish). This could suggest that my brown fat (or browning of my white fat depots) is more active in this period than before the diet. Perhaps this reflects that the body anticipate (as it would have in the stone-ages perhaps) when normal daily activity ceases and we go to sleep there would be a need for internal heating to keep us warm during the night? The experience suggest that I now have a more active brown (and/or white) fat at this time and am better able to produce internal heat than before the diet. It would therefore be interesting to measure UCP1 and other genes related to heat production in fat depots before and after some months on the diet for comparison.

\section{Study on 5:2 diet}

Unfortunately I do not have any measures apart from weight loss and performance during exercise to substantiate the impact of the diet. I do not have measurements of IGF-1, blood pressure, cholesterol or fat levels, blood glucose or insulin for that matter. To this date there are not any available scientific publications that systematically have investigated the 5:2 diet as a way to improve health (PubMed search). However, a group led by Professor Kerstin Brismar at Karolinska in Stockholm has conducted a one year study on a little over 100 obese subjects of which approximately half were also type-2 diabetics. ${ }^{2,3}$ Some of the findings from this study was reported in two TV documentaries (Swedish television; SVT) and a few news articles online (Google search). In this study all subjects experienced weight reduction and had reportedly improved values of blood glucose, fat and cholesterol as well as reduced/normalized blood pressure. The improvements were best for the diabetic group. The results strongly suggest that the 5:2 diet would be of great benefit to overweight subjects in particular diabetics and pre-diabetics. Interestingly there were very few subjects that left the study due to the diet. Compared to other diets where dropouts can be $20 \%$ or more, this was very encouraging and emphasize the idea that this diet is much easier to follow. Hopefully we will soon see the results of the Karolinska study in scientific publications and can learn from the details on weight reduction, IGF-1, cholesterol and more.

\section{Concluding remarks}

My own experience has been that the 5:2 diet provided an opportunity to accelerate my exercise amount and performance almost immediately after starting, suggesting that there were considerable hormonal-metabolic changes after a few weeks. This can be interpreted as if my energy depots became more accessible probably by improving the hormonal responses in liver and adipose tissue. I also experienced that recovery after exercise was much faster and the urge to run again came back the same day. In fact in the first half year on the diet I had many days feeling invigorated and with better general energy level very often on the fasting days. Sleep also improved in this period though occasionally on the night following a fasting day I could wake up and feel hungry and not completely at ease. Now after four years on the diet it has become a part of living and does not bother me in any way. It saves me time and money and I feel 10 years younger. Probably my physical and mental health is better and I know for sure that my body weight and fitness is much better than four years ago.

Overall these experiences together with the Karolinska study suggests that obese, overweight diabetics and pre-diabetics could benefit from this diet. In particular if combined with increase in exercise and perhaps also including good guidance on food and nutrients for some subjects. To me the diet immediately provided an opportunity to increase exercise amount and the performance improved along with the urge to do more. The combined diet and exercise it likely to improved general health as suggested in the Karolinska study. This should also encourage investigations of the diet in other labs with obesity and diabetes focus. There are many parameters and biomarkers to study and combined with interviews with enrolled subjects we could learn many details of the outcome over time.

\section{Acknowledgements}

None.

\section{Conflict of interest}

The author declares no conflict of interest.

\section{References}
1. Eat, Fast and Live Longer.
2. 5:2 hysteri - vad hände sen?
3. Kerstin Brismar: Står upp för 5:2-dieten. 